\title{
FAMILY FUNCTIONING CHARACTERISTICS INVOLVED IN ADOLESCENT DEPRESSIVE SYMPTOMS
}

\author{
Antonio Caño ${ }^{1}, \&$ Carmen Rodríguez-Naranjo ${ }^{2}$ \\ ${ }^{1}$ Department or Personality, Assessment and Psychological Treatment, Faculty of Psychology, \\ University of Málaga (Spain) \\ ${ }^{2}$ Department of Basic Psychology, Faculty of Psychology, University of Málaga (Spain)
}

\begin{abstract}
Research has shown that family functioning contributes to depressive symptoms in adolescents, with a wide range of family functioning characteristics associated to adolescent depressive symptoms. However, these family attributes have been studied through different studies, methodologies and theoretical frameworks, and do not allow envisaging a single whole picture of the family attributes associated to adolescent depressive symptoms. The objective of this study was to overcome this deficit. We followed a systematic approach and used the Family Assessment Device (FAD), which comprehensively identify six family variables in which healthy and unhealthy families differ: Problem Solving (PS), Communication (CM), Roles (RL), Affective Responsiveness (AR), Affective Involvement (AI) and Behaviour Control (BC). Independent regression analyses conducted for each variable showed that all the FAD variables significantly predicted BDI scores. However, when the six variables were introduced simultaneously in the same equation to control for the shared explained variance, only AR and AI showed significant effects, with BC approaching significance. These results were confirmed through Pratt's measure, which showed that the non-overlapping effects of $\mathrm{AR}, \mathrm{AI}$ and $\mathrm{BC}$ accounted for virtually the whole variance explained by the FAD dimensions. Conclusions at both methodological and applied levels emerge from these results. At a methodological level, these results prove the need for controlling the shared variance between family variables before deriving any conclusion about their role. At an applied level, they showed that the family affective aspects are the most important regarding adolescent depression, with only behaviour control playing a role within the non-affective variables.
\end{abstract}

Keywords: Family functioning, adolescent depression.

\section{Introduction and objectives}

Family functioning has demonstrated to be a strong risk factor for developing depressive symptoms during adolescence, which has been primarily defined by broadband variables like family cohesion, warmth, acceptance or support, which represent family as a whole (Guassi Moreira and Telzer, 2015). However, it is necessary to carry out a more fine-grained analysis of the specific family functioning variables that affect adolescent depressive symptoms and this is the main purpose of this study. With this purpose, the variables of the MacMaster Model of Family Functioning (MMFF; Miller, Ryan, Keitner, Bishop, and Epstein, 2000) were used in this study, which have been found useful in distinguishing healthy and unhealthy families in clinical and research settings. Research has shown that adolescents' global scores of the MMFF are related to their depressive symptoms (Millikan, Wamboldt, and Bihun, 2002). More recently, Rodriguez-Naranjo and Caño (2018) carried out a study to test the relationship between the variables included in the MMFF and depressive symptoms in adolescents, finding that both global scores of the MMFF and also each MMFF variable on its own predicted depressive symptoms. However, in that study it remains the possibility that the different MMFF variables predicted depressive symptoms due to its shared variance and the prediction was not a genuine effect of each single variable. To our knowledge no study has aimed to differentiate between the ability of the MMFF variables to predict depressive symptoms, once the shared variance between them have been controlled, and this is the main objective of this study. 


\section{Method}

Our sample consisted of 643 secondary-students between 12 and 17 years old. To assess the specific variables of family functioning, and its overall functioning, we used the Family Assessment Device (FAD; Epstein, Baldwin \& Bishop, 1983), including its six subscales: Problem Solving (PS), Communication (CM), Roles (RL), Affective Responsiveness (AR), Affective Involvement (AI) and Behaviour Control (BC). The Beck Depression Inventory (BDI; Beck, Ward, Mendelson, Mock, \& Erbaugh, 1961) was used to assess depressive symptoms in adolescents. We also measure socio-economic status (SES) through a six-item scale assessing the education and occupation of the parents, and housing conditions. All these scales showed good internal consistencies in our sample, with ranges of .65 to .89 .

\section{Design and results}

We conducted seven hierarchical regression equations to predict depressive symptoms. In all of them sex, age and SES were introduced in the first step for control purposes. In six of them one single MMFF variable was included in the second step, while in the seventh regression analyses all the MMFF variables were introduced jointly in the second step. When there were introduced in separate analyses, all the FAD variables significantly predicted depressive symptoms (all $p s<.001$ ). However, the results were at contrast when the six FAD variables together in the same equation, in order to control for the shared explained variance between FAD variables. Once controlled for the common variance, only AR and AI showed significant effects $(\beta=.21, p<.001$ for $\mathrm{AR} ; \beta=.16, p<.01)$, with $\mathrm{BC}$ approaching significance $(\beta=.09, p=.07)$.

Additionally, to this significance tests, Pratt's measures were calculated to identify the amount of unique variance accounted for each variable (Pratt, 1987). This is a measure aimed to study unique effects of predictors in a multiple regression analyses, and one of its important attributes is that their aggregation across the different predictors equals the overall explained variance $\left(R^{2}\right)$, thus it distributes $R^{2}$ between the different predictors avoiding any overlap between them. As a rule of thumb, predictors with Pratt's measures higher to the inverse of the number of predictors included in the regression equation are considered important (Wu, Zumbo \& Marshall, 2014). Results are presented in Table 1, showing that the non-overlapping variance accounted for $\mathrm{AR}, \mathrm{AI}$, and $\mathrm{BC}$ were above the criterion recommended to consider important a predictor (.11 for nine predictors). Actually, these three FAD dimensions accounted up to $95 \%$ of the total variance explained by the demographics and FAD dimensions.

Table 1. Unique effects of demographics and FAD dimensions for the prediction of BDI.

\begin{tabular}{|c|c|c|c|}
\hline & $\beta$ & $\begin{array}{c}\text { Cross product } \\
\beta^{*} \mathrm{r}\end{array}$ & $\begin{array}{l}\text { Pratt's measure } \\
\mathrm{d}=\left(\beta^{*} \mathrm{r}\right) / \mathrm{R} 2\end{array}$ \\
\hline Gender & .38 & .001 & .004 \\
\hline Age & -.034 & -.001 & -.005 \\
\hline SES & $-.096^{*}$ & .011 & .069 \\
\hline PS & -.025 & -.006 & -.039 \\
\hline $\mathrm{CM}$ & -.049 & -.012 & -.076 \\
\hline RL & .052 & .016 & .098 \\
\hline $\mathrm{AR}$ & $.213 * * *$ & .072 & .453 \\
\hline AI & $.159 * *$ & .051 & .322 \\
\hline \multirow[t]{2}{*}{$\mathrm{BC}$} & $.093+$ & .028 & .174 \\
\hline & & $\begin{array}{c}\sum_{\mathrm{R}^{2}} \beta^{*} \mathrm{r}= \\
\mathrm{R}^{2}=16\end{array}$ & $\sum \mathrm{d}=1$ \\
\hline
\end{tabular}

\section{Conclusions}

Conclusions at both methodological and applied levels emerge from these results. At a methodological level, these results prove the need for controlling the shared variance between predictors in regression analyses before reaching any conclusion about them. In our study the results were totally at odds depending on weather we regressed BDI scores on each FAD variable in different equations -thus without controlling its communality with other FAD variables- or we regressed BDS scores on the six variables at the same time -then controlling the shared explained variance between them. In the first case, all the FAD dimensions significantly predicted BDI scores, what did not allow revealing any 
discrimination between the different family functioning dimensions in order to predict BDI scores. However, more interesting results emerged once the shared variance between family functioning variables. Thus only two out of six (affective response, affective involvement) significantly predicted BDI scores, with behaviour control being very close to the signification. But the important point is that, in contrast to this variables, three family functioning variables (problem solving, communication and roles) were irrelevant in order to predict BDI scores in adolescents. Thus, an analytical approach that adequately attributes and distinguish unique versus shared effects between predictors is required to allow a fine-grained analysis of the specific family functioning variables that affect adolescent depressive symptoms.

At an applied level, our results show that the family affective aspects are the most important regarding adolescent depression, with only behaviour control playing a role within the non-affective variables. The importance of affective aspects within the family in order to prevent depressive symptoms are in line with other results that shown the importance of emotional bonding between family members to foster adolescents' emotional regulation and prevent depressive symptoms in adolescents (Yap, Allen, \& Sheeber, 2007). Of particular importance is the finding that adolescent perceptions of behavioural control also contributed to their depressive symptoms, which is consistent with other findings for parental monitoring (e.g., Kim \& Ge, 2000). Yap et al. (2007) argue that affective and behavioural impulses raise in adolescence simultaneously to a decrease of external monitoring, thus explaining heightened vulnerability to emotional problems at these ages. Our findings support this and suggest that both family affection and behavioural control are instrumental in promoting adolescents' emotional regulation in response to developmental challenges.

\section{References}

Miller, I. W., Ryan, C. E., Keitner, G. I., Bishop, D. S., \& Epstein, N. B. (2000). The McMaster Approach to Families: Theory, assessment, treatment and research. Journal of Family Therapy, 22(2), 168-189.

Millikan, E., Wamboldt, M. Z., and Bihun, J. T. (2002). Perceptions of the family, personality characteristics, and adolescent internalizing symptoms. Journal of the American Academy of Child \& Adolescent Psychiatry, 41, 1486-1494.

Rodríguez-Naranjo \& Caño, A. (2018). Family functioning, socio-economic status and adolescents' depressive symptoms: the mediating role of hopelessness. In C. Pracana and M. Wang (Eds). Psychological Applications and Trends (308-310). Lisbon: InSciencePress.

Epstein, N.B., Baldwin, L., \& Bishop, D.S. (1983). The McMaster Family Assessment Device. Journal of Marital and Family Therapy 9: 171-180.

Beck, A. T., Ward, C. H., Mendelson, M., Mock, J., \& Erbaugh, J. (1961). An inventory for measuring depression. Archives of General Psychiatry, 4, 561-571.

Pratt, J. W. (1987). Dividing the indivisible: Using simple symmetry to partition variance explained. In T. Pukilla \& S. Duntaneu (Eds.), Proceedings of Second Tampere Conference in Statistics (pp. 245-260). Tampere, Finland: University of Tampere.

Wu, A. D., Zumbo, B. D., \& Marshall, S. K. (2014). A method to aid in the interpretation of EFA results: An application of Pratt's measures. International Journal of Behavioral Development, 38 (1), 98-110.

Yap, M.B.H, Allen, N.B., and Sheeber, A.L. (2007). Using an emotion regulation framework to understand the role of temperament and family processes in risk for adolescent depressive disorders. Clin. Child Family Psychol. Rev., 10,180-196.

Kim, S. Y., \& Ge, X. (2000). Parenting and adolescent depressive symptoms in Chinese American Families. Journal of Family Psychology, 14, 420-435. 